\title{
AN EFFECTIVE DOPPLER FILTERING USING HIGHER-ORDER STATISTICS
}

\author{
Boryana VASSILEVA
}

\section{Introduction}

The moving target detection (for example of aircraft) in disturbed environment is an important radar processing problem. This task is to be performed by the pulse Doppler signal processor. The latter has two basic functions:

- to maximize the detection probability of a target signal and at the same time maintain a Constant False Alarm Rate (CFAR);

- to estimate the Doppler frequency shift, i.e. to estimate the target radial velocity.

A Doppler processor normally consists of a Reject Filter (RF) followed by a Fast Fourier Transform (FFT) and a CFAR processor. The technique for clutter suppression commonly in use today is RF based on Maximum Entropy Method (MEM) proposed by J. Burg. ${ }^{1}$ However, for low Clutter-to-Noise-Ratio (CNR) this technique is not successful. Indeed, as the Power Spectral Density (PSD) of the received signal is already relatively flat due to noise, the MEM filter will not whiten further the PSD significantly.

For the same reason the use of a MEM matched filtering is quite limited and the classical FFT technique is preferable. However FFT does not perform an effective matched filtering when the input Signal-to-Noise-Ratio (SNR) is very poor. ${ }^{2}$

To reduce the degradation of the Doppler filtering in the presence of powerful noise several approaches have been proposed. ${ }^{3}$ However, they have only moderate success. Lately, higher-order statistics are finding wider applicability in signal processing. ${ }^{4}$ In order to improve radar Doppler filtering under the mentioned condition, MEM algorithm using higher-order statistics is developed and described in this work. The performance of the proposed algorithm is investigated by means of simulation analysis. 


\section{Background}

Let the received signal $z(i)$ be an additive mix of an echosignal $y(i)$ and an independent zero-mean noise $n(i)$ with variance $\sigma_{n}^{2}$, i.e.

$$
z(i)=y(i)+n(i), \quad i=0,1, \cdots K-1 .
$$

The received signal is assumed stationary in a broad sense. Its autocorrelation function (ACF) depends only on the time difference, i.e.

$$
R_{z}(k)=R_{y}(k)+\sigma_{n}^{2} \delta(k) \quad k=0,1, \cdots K-1,
$$

where $R_{y}(k)$ is the ACF of the echosignal and $\delta(k)$ is the discrete delta function $(\delta(k)=1$ when $k=0$ and $\delta(k)=0$ when $k \neq 0$ ). The normalized ACF of the received signal is

$$
B_{z}(k)=R_{z}(k) / R_{z}(0)=\left\lfloor r B_{y}(k)+\delta(k)\right\rfloor /(r+1), \quad k=0,1, \ldots, K-1
$$

where $B_{y}(k)=R_{y}(k) / R_{y}(0)$ is the normalized ACF of the echosignal, $r$ is the Echosignal-to-Noise-Ratio (ESNR).

The $m^{\text {th }}$ order ACF and the normalized ACF are respectively

$$
\begin{aligned}
R_{z m}(k) & =\left\{\begin{array}{lrr}
z(k) & \text { if } & m=0 \\
E\left[z(i) z^{*}(i+k)\right] & \text { if } & m=1 \\
E\left[B_{z(m-1)}(i) B_{z(m-1)}^{*}\right] & \text { if } & m>1
\end{array}\right. \\
B_{z m}(k)=R_{z m}(k) / R_{z m}(0) & k=0,1, \cdots K-1 .
\end{aligned}
$$

For these functions the biased estimation

$$
\hat{R}_{z m}(k)=\sum_{i=0}^{K-k-1} \hat{B}_{z(m-1)}(i) \hat{B}_{z(m-1)}^{*}(i+k) / K
$$

is used. This estimation tends to have less mean-square error for finite data records compared with the unbiased estimation

$$
\breve{B}_{z m}=\sum_{i=0}^{K-k-1} B_{z(m-1)}(i) \breve{B}_{z(m-1)}^{*}(i+k) /(K-k) .
$$

Based on eq. (2), it is proved by means of induction that if the ESNR is not very poor the following relation is true

$$
\hat{B}_{z m}(k)-\hat{B}_{y m}(k) \sim C_{m}(r)\left(1 / K^{m-1}\right)
$$


and therefore

$$
\hat{B}_{z m}(k)=\hat{B}_{y m}(k)+O\left(1 / K^{m-1}\right), \quad k=0,1 \cdots K-1 .
$$

If $r \rightarrow \infty$ then $C_{m}(r) \rightarrow 0$ and $\hat{B}_{z m}(k)=\hat{B}_{y m}(k)+o\left(1 / K^{m-1}\right)$. Consequently, with the increase of the order of the normalized ACFs of the received signal and the echosignal, their estimations approximate each other. The greater the length of the data record is and the greater the ESNR is, the closer they get to each other. The simulation analysis that has been carried out confirms relation (8). When $r=-10 d B, \quad K=32$ and $m \geq 3$, the estimations $\hat{B}_{z m}(k)$ and $\hat{B}_{y m}(k)$ are sufficiently close to each other for practical purposes. Better results are obtained when $K=64$.

Further, given the realistic assumption, that the echosignal model is Autoregressive (AR) with coefficients $\beta(l), \quad l=1,2, \cdots p,(p \leq 5)^{3}$ the model of the received signal can be expressed in the following way

$$
z(i)=\sum_{l=1}^{p} \beta(l) z(i-l)+\sum_{l=1}^{p} \alpha(l) \varepsilon(i-l)+\varepsilon(i),
$$

where $\varepsilon(i)$ is the zero-mean white noise with variance $\sigma_{\varepsilon}^{2}$ and $\alpha(l)$ are the moving average coefficients. An important relation between the parameters of the process and its normalized ACF $B_{z m}(k)$ exists. It can be achieved as follows. Multiply (9) by $z^{*}(i+k)$, take the expectation and normalize autocorrelation lags thus obtained. If the result is multiplied by $B_{z 1}^{*}(i+k)$ and the expectation is taken again, it becomes evident that for the $m^{\text {th }}$ order normalized ACF the following relation is true

$$
B_{z m}(i)= \begin{cases}\sum_{l=1}^{p} \beta^{*}(l) B_{z m}(i-l) \text { if } m \text { is odd } & \\ \sum_{l=1}^{p} \beta(l) B_{z m}(i-l) \text { if mis even } & , i \geq p+1\end{cases}
$$

Relation (10) gives the AR model of the normalized autocorrelations, given values of the argument $i \geq p+1$. Hence, in order to estimate the parameters of this model (that in this case are the parameters or the complex conjugated parameters) of the AR model of the echosignal, the MEM can be used.

\section{Suboptimal Doppler Filtering Algorithm}

In this section suboptimal filtering algorithm for radar Doppler processing, based on the approach described in section 2 is presented. Figure 1 shows a blockdiagram of this algorithm. 


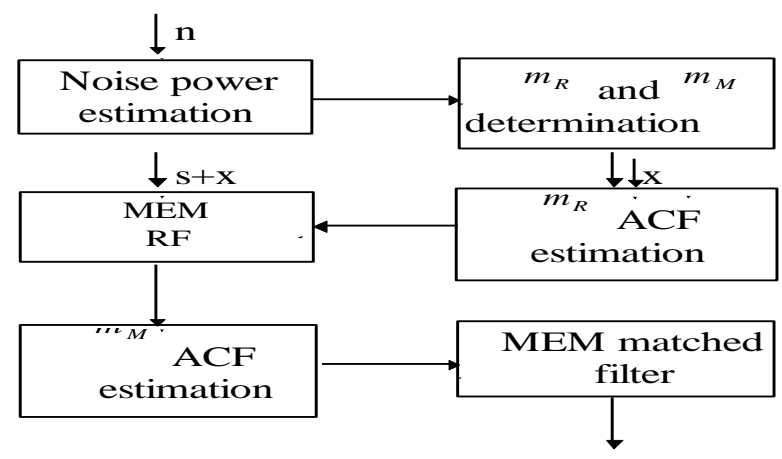

Figure 1

The received noise power is estimated in the passive operating mode of the radar. Depending on the noise power level and the power levels of the expected echosignals the orders of the statistics for the RF input $\left(m_{R}\right)$ and the matched filter input $\left(m_{M}\right)$ are determined for each range cell. The disturbing signals $x_{n}(k), n=1,2, \cdots K_{r}$ received from $K K_{r}$ range cells around the testing cell are used for estimation of the $m_{R}$-order ACF. The so obtained ACF lags $B_{n}(k), k=p+1, \cdots K$ are used to compute the RF coefficients $a(l), l=1,2, \cdots p_{R}$ implementing the multisegment Burg's recursive algorithm.

As the first step forward and backward sequences $f_{l, n}(k)$ and $b_{l, n}(k)$ are initialized as

$$
f_{1, n}(k)=B_{n}(k+1) ; b_{1, n}(k)=B_{n}(k), k=p_{R}+1, \cdots K-1, n=1,2, \cdots K_{r} .
$$

The first reflection coefficient is computed as

$$
\rho_{l}=\frac{\sum_{n=1}^{K_{r}} \sum_{k=p_{R}+1}^{K-l} 2 f_{l, n}(k) b_{l, n}^{*}(k)}{\sum_{n=1}^{K_{r}} \sum_{k=p_{R}+1}^{K-l}\left[\left|f_{l, n}(k)\right|^{2}+\left|b_{l, n}(k)\right|^{2}\right]},
$$

with $l$ equal to unity.

A recursive relation for the higher-order reflection coefficients is obtained via the update relations:

$$
\left.\begin{array}{l}
f_{l, n}(k)=f_{l-1, n}(k+1)-\rho_{l-1} b_{l-1, n}(k+1) \\
b_{l, n}(k)=b_{l-1, n}(k)-\rho_{l-1}^{*} f_{l-1, n}(k)
\end{array}\right\}, \quad k=p_{R}+1, \cdots K-l .
$$


This is continued until $l=p_{R}$. The filter coefficients $a(l)$ are then calculated from the recursive relation

$$
a_{p_{R}}(l)=a_{p_{R}-1}(l)+\rho_{l} a_{p_{R}-1}^{*}\left(p_{R}-l\right) ; \quad a_{p_{R}}(l)= \begin{cases}1 & l=0 \\ \rho_{l} & l=p_{R} \\ 0 & l>p_{R}\end{cases}
$$

For the testing cell the output of the RF is

$$
Z(i)=\left\{\begin{array}{l}
B_{z m_{R}}(i)-\sum_{l=1}^{p_{R}} a^{*}(l) B_{z m_{R}}(i-l) \text { if } m \text { is odd } \\
B_{z m_{R}}(i)-\sum_{l=1}^{p_{R}} a(l) B_{z m_{R}}(i-l) \text { if } m \text { is even }
\end{array}, \quad i \geq p_{R}+1\right.
$$

$B_{z m_{R}}(i)$ are the $m_{R}$-order normalized ACF lags for $z(i)=s(i)+x(i)$, where $s(i)$ is the useful signal and $x(i)$ is the disturbing signal. The RF output signal is used for $m_{M}$-order ACF estimation. The so obtained correlations are input data for the first-order matched filter. The output of this filter is a PSD sequence

$$
\hat{\phi}(k)=\frac{\sigma^{2}}{2 \pi\left|\sum_{n=0}^{1} \beta(n) \exp \left(-j \frac{k}{K} n\right)\right|^{2}}, \quad k=0,1, \cdots K-1
$$

where $\beta(0)=1, \beta(1)$ is calculated from (12) for $n=1, \quad l=1, \quad k=6,7, \cdots K-1$ and $\sigma^{2}$ is the prediction error power. Formula (16) is the spectral line expression for different frequencies (velocity channels).

\section{Simulation example}

The following example is used to illustrate the performance of the proposed algorithm. Let the interference be defined by the return echoes from the sea and the Stand-of-Jammer (SOJ) broadcasting wideband noise. Amplitude responses of the first-order RF for $m_{R}=1$ (RF1) and for $m_{R}=2$ (RF2) are shown in Figure 2. The numbers of the velocity channels are given along the abscise. The radial velocity of the clutter is determined by the $0^{\text {th }}$ channel. The CNR is $-3.5 \mathrm{~dB}$. The filters coefficients are estimated for $K=64$ and $K_{R}=2$. The curves show better clutter rejection for RF2. Besides, the significant pass band irregularity of RF1 can distort undesirably the useful signal. 


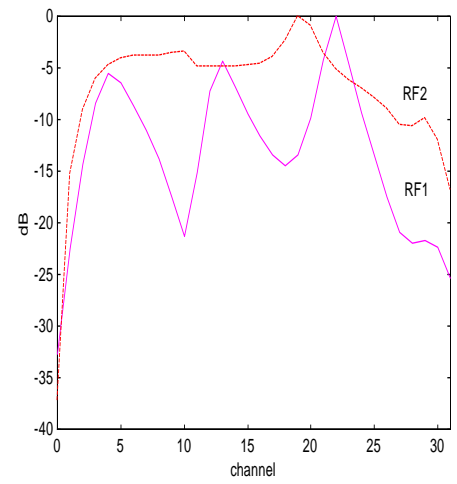

Figure 2

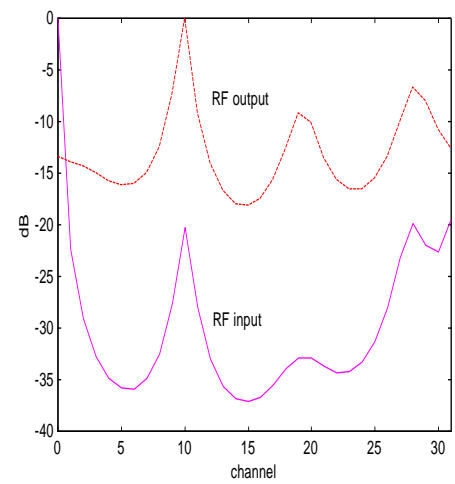

Figure 3

Therefore, RF2 is the preferable filter. Figure 3 presents normalized spectral lines of the interference second-order ACF before and after rejection. We can see the RF2 whitening operation.

To investigate the performance of the complete algorithm the useful signal is added to the considered interference. The SNR is $-10.5 d B$. The Signal-to-Clutter-Ratio (SCR) is $-5.5 d B$. The radial velocity of the target (slowly flying airplane) is determined by the second-channel. The line "FFT" on Figure 4 shows the received signal FFT spectrum, which is relatively flat. The "MEM"- line shows the tenth-order MEM spectrum of the second-order signal ACF. Estimation of the echosignals peaks with high resolution is achieved.

The results of the complete algorithm for $m_{R}=2, m_{M}=2$ and $p=1$ are shown on Figure 5. The line "Target" shows the selected useful signal spectrum and the line "Noise" shows the residual noise spectrum. The output SNR is more than $20 \mathrm{~dB}$. In spite of nearness of the clutter and target velocity channels, the Improvement Factor (IF) is more than $30 \mathrm{~dB}$. The so obtained IF value ensures high quality of signal detection. 


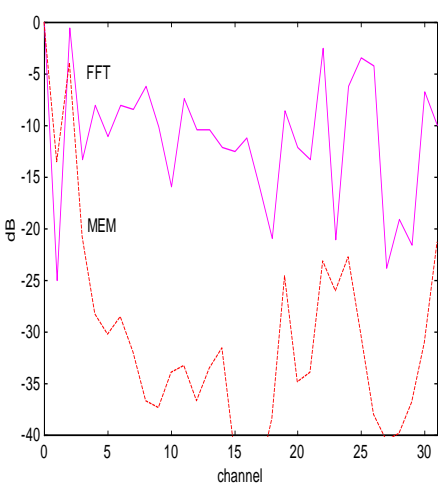

Figure 4

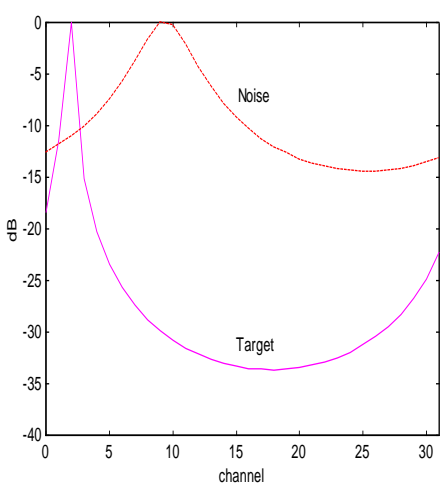

Figure 5

\section{Conclusions}

In this paper a new algorithm for moving target signal selection in the presence of clutter and wideband jammer is presented. The proposed algorithm includes whitening filter for the interference, followed by a matched filter for the useful signal. This cascade of filters is based on higher-order statistics approach (namely higherorder ACF) and maximum entropy pole estimations. The structure of the described algorithm is relatively simple. It is appropriate for on-line processing. The algorithm performance is investigated by means of the Monte Carlo simulation analysis. The results indicate that the proposed algorithm is very effective particularly for short data records. The obtained frequency resolution and IF values ensure high quality of signal detection.

It should be noticed that for relatively long data records the use of cumulants as a higher-order statistics and maximum entropy pole-zero estimations for radar Doppler filtering is a very promising approach. Future work will focus on developing such a type of adaptive algorithm.

\section{Acknowledgement}

The work on this paper was partially supported by the Bulgarian National Foundation for Scientific Investigations under grant I-808/98.

\section{References}

1. J.P. Burg, Maximum Entropy Spectral Analysis (Stanford University, 1967), 1-32.

2. Boryana Vassileva, "High Resolution Matched Filtering in Radar Doppler Processor," Comptes rendus de l'Academie bulgare des Sciences 52, 4 (1999, to appear). 
3. S.M. Kay and S. L. Marple, Spectrum Analysis. A Modern Perspective, IEEE, vol. 69, 11 (1981), 1380-1419.

4. J.M. Mendel, "Tutorial on Higher-Order Statistics (Spectra) in Signal Processing and System Theory: Theoretical Results and Some Applications," IEEE 79, 3 (1991), 278305.

5. S. Haykin, B. W. Currie and S. B. Kesler, "Maximum Entropy Spectral Analysis of Radar Clutter," IEEE 70, 9 (1982), 51-62.

BORYANA PETROVA VASSILEVA is assistant research professor at the Central Laboratory for Parallel Processing, Bulgarian Academy of Sciences. She received M.S. in University of Byelorussia in Minsk in 1975 and Ph.D. degree in Sofia Technical University in 1999. E-mail: signal@bas.bg. 